\title{
Multi-period Robust Portfolio Selection Model with Bat Algorithm
}

\author{
Xing Yu \\ Department of Mathematics \& Applied Mathematics, Hunan University of \\ Humanities, Science and Technology, Loudi, 417000, P.R. China \\ hnyuxing@163.com
}

\begin{abstract}
This paper proposes a portfolio selection model in which the methodologies of robust optimization are used for the maximization of the terminate wealth under the constraints of each stage risk described as absolute deviation of not more than the given levels. In place of stochastic programming, it is used of techniques of robust optimization to deal with uncertainty. Moreover, we succeeded in solving the multi-period portfolio selection model with use of the bat algorithm (BA). Numerical results show that there are two important features of our work. One is that the yield curve is smooth, less fluctuation. So, it is of less psychological impact on investors, cash flow smooth. Another, BA applied to solve the model is feasible and it is more effective than genetic algorithm.
\end{abstract}

Keywords: Absolute deviation; Robust optimization; Bat algorithm; Portfolio selection

\section{Introduction}

The goal of portfolio theory is how to allocate a certain amount of money in different assets in an optimal way. And the portfolio theory is the foundation of modern financial investment. Markowitz's pioneered work is to propose the classical mean-variance(MV) model for portfolio selection problem and risk management. Since Markowitz framework, there is increasing research on this subject in order to provide the portfolio selection model more realistic conditions. On direction is that a drawback of MV model is tuned to a single period. In fact, an investor will adjust invest strategy according to the renewed information dynamically. To overcome this issue, one way formulate from the beginning the allocation problem over a horizon composed of multiple periods. The extensive literates about this direction can be seen, for instance, $\mathrm{Li}$ and $\mathrm{Ng}$ introduced a technique to tackle the multi-period mean-variance problem, with market uncertainties reproduced by stochastic models, in which the key parameters, expected return and volatility, are deterministic. Yong proposed a multi-stage mean-variance portfolio selection model by using interval analysis. The goal of the related literates either is to minimize the total or terminate risk of the investment path while satisfying constraints on the portfolio composition and on desired expected return either to maximize terminate wealth under the constraint of the end risk, what is more, the expected value of the portfolio return is maximized simultaneously with the minimization of its variance. And according to the actual situation, at the same time to achieve the goal of minimizing the risk of revenue is very difficult to achieve. Secondly, for an investor, the purpose is to get the benefits, but not the least risk. Rather than pursuing the least risk, it is better to make money without risk investment, such as in the bank. Of course, to maximize the benefits of the control risk is more realistic. Because a lot of literature does not consider the investment process control, that is to make the final risk less than the default constraint. But if the volatility of the investment process is too large, for investors, the psychological impact is very large. Even after a long loss, the investment will be forced to terminate. So our goal is to obtain make the maximum income meanwhile constraint each stage risk. Another weak point of 
MV model is that the parameters are assumed to be certain according to the history data. But it is a fact that we should make decision for the future, but future is not be replaced by history, and the future is uncertain. So the optimization process leads to solutions heavily on the parameters perturbations. In this context, we take account in uncertainty in optimization model and make worst estimations about the unknown information which is so-called robust optimization technique. Anna deals with a portfolio selection model in which the methodologies of robust optimization are used for the minimization of the conditional value at risk of a portfolio of shares. Shapiro considers the adjustable robust approach to multistage optimization, for which we derive dynamic programming equations.

The rest of the paper is organized as follows. Section 2 describes the multi-period mean deviation optimization problem. In section 3 , we introduce robust framework of the model. In section 4 the BA is presented. Section 5 presents the numerical example and results to show the practically and efficiency of our model and algorithm. Finally, section 6 conclusions the paper.

\section{Multi-Period Mean Deviation Portfolio Model}

We consider there are $n$ risk assets in the financial market. A given finite investment horizon is $T$, which is divided by equal duration $\Delta, \Delta$ is a fixed time span. For instance, if we let $\Delta=1$, it means one month, and it can mean one year also, for example. After the initial investment at $\mathrm{t}=0$, the portfolio may be restructured at the beginning of period $t(t=1,2 \cdots T-1)$. Suppose that $x_{i}(t)$ is the portion of the investor's total wealth invested in asset $i(i=1,2 \cdots n)$ at the end of period $t$. The investor's total amount at the end of period $t$ is given by $\sum_{i=1}^{n} x_{i}(t)$.

At the beginning of each period, the investor may adjust the portfolio composition. We denote with $x_{i}(t+1)$ the portfolio composition just after the adjust $a_{i}(t)$ occurred at the beginning of period $t+1$. So $x_{i}(t)=x_{i}(t-1)+a_{i}(t)$. if $a_{i}(t)>0$, it means that the portfolio content in asset $i$ is deceased by $a_{i}(t)$ or selling this asset. Similarly, if $a_{i}(t)<0$, it means that the portfolio content in asset $i$ is increased by $a_{i}(t)$ or buying this asset. So the total amount of transaction is $a(t)=\sum_{i=1}^{n} a_{i}(t)$. We assume that the portfolio is a self-financing portfolio, that is, $a(t)=0$ in this paper. And suppose that $p_{i}(t)$ denotes the market value of asset $i$ at the end of period $t$, the simple return of an investment over period $[t-1, t]$ is defined as

$$
\begin{gathered}
\begin{aligned}
r_{i}(t) & =\frac{p_{i}(t)-p_{i}(t-1)}{p_{i}(t-1)} \\
& =\frac{p_{i}(t)}{p_{i}(t-1)}-1
\end{aligned} \\
\text { So } p_{i}(t)=\left(1+r_{i}(t)\right) p_{i}(t-1)
\end{gathered}
$$


The value of the portfolio at $t=0$ is $W_{0}=\sum_{i=1}^{n} x_{i}(0) p_{i}(0)$. If we suppose at the beginning investors have an unit wealth, that $W_{0}=1$, and the price of each asset in the beginning are known as $p_{i}(0)$.

At $t=1$, the value of the portfolio is

$$
W_{1}=\sum_{i=1}^{n} x_{i}(1) p_{i}(1)=\sum_{i=1}^{n}\left(x_{i}(0)+a_{i}(1)\right) p_{i}(0)\left(1+r_{i}(1)\right)
$$

We can obtain the value of the portfolio at any stage $t$ :

$$
W_{t}=\sum_{i=1}^{n}\left(x_{i}(0)+\sum_{l=1}^{t} a_{i}(l)\right) p_{i}(0) \prod_{k=1}^{t}\left(1+r_{i}(k)\right)
$$

Continue to do so, invested at the end, the total wealth portfolio is

$$
W_{T}=\sum_{i=1}^{n}\left(x_{i}(0)+\sum_{l=1}^{T} a_{i}(l)\right) p_{i}(0) \prod_{k=1}^{T}\left(1+r_{i}(k)\right)
$$

According to the theory of dynamic mean semi-variance criterion, the investor seeks an optimal investment strategy to maximize the terminate wealth under the constraints of each stage risk not less than the expected value. Here, the risk we define is the difference between the expected return.

It is shown as follows

$$
\begin{gathered}
\left(W_{t}-d_{t}\right)^{-} \leq \rho_{t} \\
\left(W_{t}-d_{t}\right)^{-}=\left\{\begin{array}{c}
0, W_{t}>d_{t} \\
d_{t}-W_{t}, W_{t} \leq d_{t}
\end{array}\right.
\end{gathered}
$$

As above discussion mentioned, we can get the following optimization model:

$$
\left\{\begin{array}{c}
\max _{x, a} W_{T} \\
\text { s.t. }\left(W_{t}-d_{t}\right)^{-} \leq \rho_{t} \\
\sum_{i=1}^{n} a_{i}(t)=0 \\
\sum_{i=1}^{n}\left(x_{i}(0) p_{i}(0)\right)=1
\end{array}\right.
$$

\section{Robust Framework of the Model}

In order to disambiguate the investment decisions, robust optimization adopts a worstcase perspective. In this modeling framework, the return vectors $r_{i}(k)$ at stage $k$ are unknown, but they are believed to change within an uncertainty set $\Theta_{r_{i}}$. In order to immunize the portfolio selection against the uncertainty of $r_{i}$, we maximize the worstcase portfolio return, where the worst-case is calculated with respect to all asset returns in $\Theta_{r_{i}}$, which is formulated as a max-min problem

$$
\max _{x, a}\left\{\min _{r_{i} \in \Theta_{r_{i}}} W_{T}\right\}
$$

In order to transfer the objective simply, we introduce 


$$
\begin{gathered}
\phi_{i}(t)=x_{i}(0)+\sum_{l=1}^{t} a_{i}(l), \\
R_{i}(t)=p_{i}(0) \prod_{k=1}^{t}\left(1+r_{i}(k)\right)
\end{gathered}
$$

So $W_{t}=\phi^{\prime}(t) R(t)$, and $W_{T}=\phi^{\prime}(T) R(T)$

Then the model can be transferred to the following formulation:

$$
\left\{\begin{array}{c}
\max _{x(0), a} \phi^{\prime}(T) R(T) \\
\text { s.t. }\left(\phi^{\prime}(t) R(t)-d_{t}\right)^{-} \leq \rho_{t} \\
a^{\prime} e=0 \\
\sum_{i=1}^{n}\left(x_{i}(0) p_{i}(0)\right)=1
\end{array}\right.
$$

There are multiple ways to specify $\Theta_{r_{i}}$. A simple choice is to use an ellipsoidal uncertainty set $\Theta_{r_{i}}=\left\{r_{i}:\left(r_{i}-\mu_{i}\right)^{T} \Sigma^{-1}\left(r_{i}-\mu_{i}\right) \leq \delta^{2}\right\}$

where $\delta=\sqrt{\frac{p}{1-p}}, p \in[0,1)$ and $\delta=+\infty$ for $p=1$.

The investor controls the size of the uncertainty set by choosing the parameter $p$ to satisfy the inequality

$$
P\left\{y^{\prime} w \geq \min _{r} y^{\prime} w\right\} \geq p
$$

\section{Bat Algorithm}

In recent years, our country launched the bat algorithm based on a series of application in all fields. Xiao-hua Sheng studied for the new heuristic intelligent algorithm bat algorithm of the limitation of discrete production scheduling problem, use the bat algorithm recording and initialization approach to solving discrete production scheduling problem, based on the classical scheduling benchmark data to test, and compared to more mature standard particle swarm optimization (pso) algorithm, the results show that the bat algorithm in solving the problem of discrete production scheduling has better optimization performance, bat is verified the feasibility and effectiveness of the algorithm to solve the discrete problem. Xin-She Yang As far as we know, there is little research on the application of bat algorithm to solve the problem of multi period portfolio investment. $\mathrm{Yu}$ proposed the mean-dynamic VaR multi-period portfolio selection model with the transaction costs and the constraints on trade volumes. The Bat algorithm is applied to solve the multi-period mean-dynamic VaR model.

Bat algorithm of the basic definition, the echo-location in bats is ideal, can be summarized as follows:

Each virtual bat has random speed of $v_{i}$ in position $x_{i}$ (solving), at the same time the bat with different frequency or wavelength, loudness, $A_{i}$ and $r$ pulse firing rate. Bats hunting and found its prey, it changes, loudness, and emissivity of the pulse frequency, the choice of optimal solution, until the target to stop or conditions are met. This is essentially a tuning technique was used to control the dynamic behavior of the bat group, balance adjustment algorithm related parameters, in order to obtain the bat algorithm of the optimal. 
According to Yang, the new solution speed $x_{i}^{t}$ and $v_{i}^{t}$ The update equation:

$$
\begin{gathered}
f_{i}=f_{\text {min }}+\left(f_{\max }-f_{\min }\right) \beta, \\
v_{i}^{t}=v_{i}^{t}+\left(x_{i}^{t-1}-x_{*}\right) f_{i}, \\
x_{i}^{t}=x_{i}^{t-1}+v_{i}^{t},
\end{gathered}
$$

Among them, the number is evenly distributed. $A$ and $r$ should be in the iterative transformation:

$$
\begin{gathered}
A_{i}^{t+1}=\alpha A_{i}^{t}, \\
r_{i}^{t+1}=r_{i}^{0}[1-\exp (-\varkappa t)],
\end{gathered}
$$

Among them, $0<\alpha<1$ and $\gamma>0$ that are constant.

\section{Numerical Example and Compared Results}

In this part, it focuses on an empirical research of an optimal portfolio model with five risk assets in Chinese market who show a good momentum in 2014. We choose the day data of four stocks NO 002583, 600694, 600089, and 601166 from 2014-01-04 09:01:00

\begin{tabular}{|c|c|c|c|c|}
\hline Stage stocks & S1 & $\mathrm{S} 2$ & S3 & S4 \\
\hline 1 & 5.50 & 2.98 & 3.54 & 4.29 \\
\hline 2 & 5.14 & 4.19 & 3.84 & 4,48 \\
\hline 3 & 4.83 & 3.35 & 2.55 & 4.29 \\
\hline 4 & 5.4 & 3.95 & 1.76 & 1.97 \\
\hline 5 & 3.32 & 4.4 & 3.31 & 285 \\
\hline
\end{tabular}
to 2015-4-1 15:00:00, which is from CSMAR database in China. But for simply, we construct the optimal portfolio model only suppose a stage conclude 5 moths. That is, there are 5 stages. The returns of these five stocks multiplied by 100 at the five stages are as follows:

Suppose $d_{t}=0.13, \rho_{t}=0.05, p=0.5$, the covariance matrix is a constant matrix as

$$
100 \Sigma^{2}=\left(\begin{array}{cccc}
2.22 & 0.27 & 0.14 & 0.35 \\
0.27 & 1.97 & 0.53 & 0.42 \\
0.14 & 0.53 & 1.62 & 0.41 \\
0.35 & 0.42 & 0.41 & 2.8
\end{array}\right)
$$

By solving the model the dynamic portfolio is

At stage $1, a_{1}(1)=0.141, a_{2}(1)=-0.107, a_{3}(1)=0.06, a_{4}(1)=0.078$;

At stage $2, a_{1}(2)=-0.082, a_{2}(2)=-0.166, a_{3}(2)=-0.059, a_{4}(2)=0.136$;

At stage 3, $a_{1}(3)=0, a_{2}(3)=0, a_{3}(3)=0.027, a_{4}(3)=-0.03$;

At stage $4, a_{1}(4)=0.063, a_{2}(4)=0, a_{3}(4)=0.047, a_{4}(4)=0.015$

and at stage $5, a_{1}(5)=-0.122, a_{2}(5)=0.077, a_{3}(5)=0, a_{4}(5)=0.102$

\section{Conclusions}

There are two main contributions of this paper. Firstly, we consider the constraints of each stage risk described as absolute deviation of not more than the given levels, which will protect the investor from the large psychological impact. Secondly, because of 
uncertain parameters, we propose the robust optimal portfolio model. Thirdly, we introduce the bat algorithm to solve the model and it is effective.

\section{Acknowledgments}

This research is supported by Natural Science Fund of Hunan (No.2016JJ6046).

\section{References}

[1] D. Li and W. L. Ng, "Optimal dynamic portfolio selection: Multi-period mean-variance formulation", Mathematical Finance, vol.10, (2000), pp.387-406.

[2] Y.J. Liu, G.Z. Wei and Z. Pu, "A multi-period portfolio selection optimization model bu using interval analysis", Economic Modelling, vol. 33, (2013), pp. 113-119.

[3] G.Q. Anna and Z. Alberto, "Robust optimization of conditional value at risk and portfolio selection”, Journal of Banking \& Finance, vol.30, no.32, (2008), pp.2046-2056.

[4] A. Shapiro, "A dynamic programming approach to adjustable robust optimization", Operations research letters, vol.39, (2011), pp.83-87.

[5] X. S., S. Deb and S. Fong, "Accelerated particle swarm optimization and support vector machine for business optimization and applications", Communications in Computer and Information Science, vol.136, (2011), pp. 53-66

[6] X.-S. Yang, "Bat Algorithm for Multi-objective Optimization", International Journal of BioInspired Computation, vol. 58, (2011), pp.287-308.

[7] X. Yu, "Multi-period Mean-dynamic VaR Optimal Portfolio Selection: Model and Algorithm", The Open Automation and Control Systems Journal, vol.7, (2015), pp. 366-369.

\section{Author}

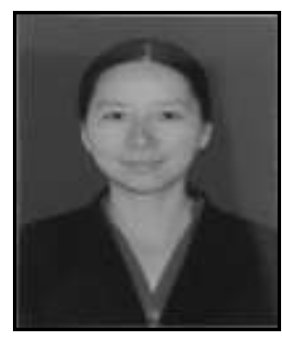

Xing Yu, she was born on February 15, 1981, in Xianning City of Hubei province. Her current position is Hunan University of humanities, Science and technology (China); Associate professor. Her professional interests are applied mathematics and Finance model. Her research interests: the optimal portfolio model; mathematical model. 